\title{
EDITORIAL
}

\section{Cognitive/Clinical Endpoints for Pre-Dementia AD Trials}

\author{
P.S. Aisen \\ Department of Neuroscience, University of California San Diego, La Jolla, CA, USA \\ Corresponding Author: Paul S. Aisen, Department of Neuroscience, University of California San Diego, La Jolla, CA, USA, paisen@ucsd.edu

$\mathbf{W}$ ith the aging of the world's population, the prevalence of Alzheimer's disease (AD) is rising. Yet it remains the only leading cause of death for which there is no disease-modifying treatment available, despite substantial academic and industry efforts. Disappointing clinical trials over the last several years have led to a growing consensus on the need to intervene early in the disease process, before clinical symptoms begin (1). Built on the hypothetical model of disease progression proposed by Jack et al $(2,3)$, which has since been supported by empirical data (4), the dominant paradigm today posits that the pathophysiologic processes underlying $\mathrm{AD}$ begin long before symptoms (5). However drug development at this stage is complicated by the difficulty of assessing a therapeutic benefit in subjects who are, by definition, clinically normal.

\section{The challenge of drug development for early stage AD}

During the dementia stages of the disease, the U.S. Food and Drug Administration (FDA) requires the demonstration of efficacy using both a measure of cognition and a functional or global assessment. This approach has worked well for the development of cognitive enhancers in mild-to-moderate stages of disease (6). The Alzheimer's Disease Assessment Scale-cognitive (ADAS $\operatorname{cog}$ ) has been the most widely used scale to capture changes in cognition (7); while global measures such as the Clinician Interview-Based Impression of Change plus Carer Interview (CIBIC-plus) and the Alzheimer's Disease Cooperative Study-Clinical Global Impression of Change (ADCS-CGIC) $(8,9)$, or the Clinical Dementia Rating Scale-Sum of Boxes (CDR-SB) (10) demonstrate that these changes are clinically meaningful.

However, these measures fall short in diseasemodifying drug development, where there is likely to be no short term symptomatic benefit but rather a change in the slope of decline. Moreover, in patients with mildto-moderate $\mathrm{AD}$ dementia, slow decline in the placebo groups means that to see a treatment effect, hundreds of subjects must be followed for at least 18 months. In even milder patients, e.g., those in the mild cognitive impairment (MCI) stage of the disease, these challenges are magnified. In addition, while some pre-dementia trials have included MCI populations, the FDA and European Medicines Agency (EMA) have never accepted $\mathrm{MCI}$ as a treatable entity for drug development. Therefore pre-dementia trials have typically used time-to-dementia as an outcome, since dementia is a treatable entity. Problems with this approach relate to subject selection, variability in conversion rate, the subjective nature of the endpoint, and artificiality of the distinction between MCI and mild AD.

These factors combine to make it nearly impossible to demonstrate proof of efficacy in a phase II-type trial. As a result, sponsors have initiated phase III studies in the absence of a clear efficacy signal. Thus far, all phase III trials of potential disease-modifying drugs have been negative. In 2013, the FDA issued a draft guidance for drug development in early stage disease (11), which recognized the challenge of demonstrating both cognitive and functional benefits prior to the onset of dementia and proposed several alternative strategies that may enable a sponsor to demonstrate a clinical benefit resulting from disease modification.

Even more important than these methodologic challenges in trial design, the failure of recent trials to demonstrate efficacy may reflect the fact that mild $\mathrm{AD}$ or even $\mathrm{MCI}$ may be too late for effective intervention, especially if targeting amyloid. The Australian Imaging, Biomarkers and Lifestyle (AIBL) study of aging, for example, showed that amyloid deposition precedes AD dementia by 15 years (12). This study, and others have led the field to move increasingly into even earlier stages of disease, before the emergence of clinical symptoms (1).

\section{Better pre-dementia designs}

Both the FDA and European Medicines Agency (EMA) have signaled a willingness to accept the concept of prodromal AD as described by the Dubois criteria (13), operationalized as MCI plus low CSF A $\beta 42$ or increased brain amyloid assessed by positron emission tomography (PET) imaging. This definition allows sponsors to 
abandon time-to-dementia as an outcome measure, replacing it with a continuous measure such as CDR-SB to capture the effect on primary manifestations of the disease and establish clinical relevance. This approach has more power than traditional MCI design (14) and is currently being used in large trials of anti-amyloid interventions in prodromal AD.

However, since the CDR-SB lacks sensitivity in very early disease stages, questions remain about the appropriate measure to assess change during the preclinical phase of disease, i.e., the population that is clinically normal but harbors AD pathology. Jack's hypothetical model of disease progression describes a sequence of changes starting with amyloid, followed by tau abnormalities and neuronal injury, then changes in brain structure, and eventually changes in memory and function. This theoretical model has proven extremely useful but is a simplification. Data accumulated from the Alzheimer's Disease Neuroimaging Initiative (ADNI) $(2,15)$, the Dominantly Inherited Alzheimer's Network (DIAN) (4), and other studies have begun to flesh out the Jack model, revealing a more complex picture. The orderly s-shaped curves of the Jack model, rather than occurring sequentially, overlap one another. While increased amyloid is the first sign to appear, it is followed by a collection of overlapping changes including tau abnormalities, cognitive changes, and functional changes. Moreover, sensitive cognitive tools begin to show abnormalities at roughly the same time as early measures of amyloid deposition.

\section{Cognition may be the best measure in prodromal populations}

Several studies have demonstrated early changes in cognition, even using standard tools such as the minimental state exam (MMSE). For example, the prospective, population-based Personnes Agées QUID (PAQUID) study in France (16), which followed people for 22 years, showed that MMSE scores begin to decline about 15 years before symptoms of disease, while global functional abilities begin to decline about 7 years prior to dementia onset (17). Cross-sectional data from populations with autosomal dominant AD have also demonstrated changes in cognition 15 years before disease onset (4).

What this means is that cognition may be a sensitive and appropriate measure of early decline that could be used in place of imaging or CSF biomarkers. While on first blush, this appears counterintuitive since by definition, people with prodromal AD are clinically normal, it now appears that there are early, subtle, and measurable changes in cognitive domains that may differentiate people with normal aging from those with AD pathology. Moreover, cognition as a measure has face validity since it is the domain that characterizes dementia and since we know which way it will move as the disease progresses.
To detect cognitive changes in preclinical AD, composite measures have been developed $(18,19)$. The ADCS developed one such composite, the ADCS Preclinical Alzheimer's Cognitive Composite (ADCSPACC) specifically for use in the upcoming Anti-Amyloid Treatment in Asymptomatic Alzheimer's (A4) study. The selection of measures for the ADCS-PACC was based on previous research suggesting the importance of assessing cognition across three key domains: episodic memory, executive function, and orientation. Thus, the ADCS-PACC incorporates a global measure of cognition (MMSE) with the Free and Cued Selective Reminding Test (FCSRT), Delayed Paragraph Recall, and the DigitSymbol Substitution Test. A recent report by the A4 investigators in cognitively normal elderly participants participating in cohort studies demonstrated that the ADCS-PACC enabled the separation of subjects with and without amyloid, as well as the separation of progressors from non-progressors and APOE4 positives from E4 negatives (20).

For regulators to accept cognition as a primary endpoint in trials enrolling a clinically normal population, they will want proof that these changes are clinically meaningful. Patient or caregiver reports of clinical or functional benefit are approaches that have been endorsed by the FDA (21). The Cognitive Function Index is one such measure, which assesses subjective impression of memory change (22). This measure is a key secondary outcome measure in the A4 trial.

Indeed, recent research suggests that cognition mediates functional decline, with most change in function related to cognition and not the reverse. Liu-Seifert et al, for example, examined data from mild AD subjects enrolled in two Phase 3 studies of solanezumab. Their analyses concluded that $87 \%$ of the treatment effect on function was attributable to improved cognition, with only $13 \%$ attributable to a direct effect on function (23). These findings strengthen the idea that a cognitive treatment signal in an early stage trial may predict later functional benefit.

\section{Conclusions}

$\mathrm{AD}$ is a gradually progressive disease, and as with other progressive diseases, intervention at the earliest possible stages is likely to have the best chance of success. Recent revisions to the diagnostic criteria have provided the field with the constructs of prodromal and preclinical disease, which more specifically reflect AD stages compared to MCI.

Recent research suggests that cognition may be the best disease single marker, capable of capturing both primary symptoms and clinically meaningful change as the disease progresses or in response to treatment. However, much work still needs to be done to validate the use of cognitive measures as primary endpoints in prodromal clinical trials. Measurement challenges represent part 
of the difficulty, and performance measures may be a significant step forward. Moreover, we should be probing cognition in terms of its components and its relationship to underlying disease and clinically meaningful change. Including new cognitive probes may result in a better understanding of early disease trajectory as well as the relationship of specific measures to clinically meaningful symptoms and signs.

Disclosures: The author has served as a consultant to the following companies: NeuroPhage, Elan, Eisai, Bristol-Myers Squibb, Eli Lilly, Merck, Roche, Amgen, Genentech, Abbott, Pfizer, Novartis, AstraZeneca, Janssen, Medivation, Ichor, Toyama, Lundbeck, Biogen Idec, iPerian, Probiodrug, Somaxon, Biotie, Cardeus, Anavex, Abbvie, Cohbar.

Acknowledgments: This work is supported by grants (NIA U01-AG10483, NIA U01-AG024904) from the National Institute on Aging of the National Institutes of Health.

\section{References}

1. Sperling RA, Jack CR, Jr., Aisen PS. Testing the right target and right drug at the right stage. Sci Transl Med. 2011;3(111):111cm33.

2. Jack CR, Jr., Knopman DS, Jagust WJ, Petersen RC, Weiner MW, Aisen PS, et al. Tracking pathophysiological processes in Alzheimer's disease: an updated hypothetical model of dynamic biomarkers. Lancet Neurol. 2013;12(2):207-16.

3. Jack CR, Jr., Knopman DS, Jagust WJ, Shaw LM, Aisen PS, Weiner MW, et al Hypothetical model of dynamic biomarkers of the Alzheimer's pathological cascade. Lancet Neurol. 2010;9(1):119-28.

4. Bateman RJ, Xiong C, Benzinger TLS, Fagan AM, Goate A, Fox NC, et al. Clinical, cognitive, and biomarker changes in the Dominantly Inherited Alzheimer Network. N Engl J Med. 2012;367(9):795-804.

5. Villemagne VL, Burnham S, Bourgeat P, Brown B, Ellis KA, Salvado O, et al. Amyloid beta deposition, neurodegeneration, and cognitive decline in sporadic Alzheimer's disease: a prospective cohort study. Lancet Neurol. 2013;12(4):357-67.

6. Rogers SL, Doody RS, Mohs RC, Friedhoff LT. Donepezil improves cognition and global function in Alzheimer disease: a 15-week, doubleblind, placebo-controlled study. Donepezil Study Group. Arch Intern Med. 1998;158(9):1021-31.

7. Mohs RC, Rosen WG, Davis KL. The Alzheimer's disease assessment scale: an instrument for assessing treatment efficacy. Psychopharmacol Bull. 1983;19(3):448-50.

8. Schneider LS, Olin JT. Clinical global impressions in Alzheimer's clinical trials. Int Psychogeriatr. 1996;8(2):277-88; discussion 88-90.

9. Schneider LS, Olin JT, Doody RS, Clark CM, Morris JC, Reisberg B, et al. Validity and reliability of the Alzheimer's Disease Cooperative StudyClinical Global Impression of Change. The Alzheimer's Disease Cooperative
Study. Alzheimer Dis Assoc Disord. 1997;11 Suppl 2:S22-32.

10. Williams MM, Storandt M, Roe CM, Morris JC. Progression of Alzheimer's disease as measured by Clinical Dementia Rating Sum of Boxes scores. Alzheimers Dement. 2013;9(1 Suppl):S39-44.

11. Food and Drug Administration. Draft Guidance for Industry. Alzheimer's disease: Developing drugs for the treatment of early stage disease. Accessed 2-24-2013 at http://www.fda.gov/downloads/Drugs / GuidanceComplianceRegulatoryInformation/Guidances/UCM338287.pdf; 2013

12. Rowe CC, Ellis KA, Rimajova M, Bourgeat P, Pike KE, Jones G, et al. Amyloid imaging results from the Australian Imaging, Biomarkers and Lifestyle (AIBL) study of aging. Neurobiol Aging. 2010;31(8):1275-83.

13. Dubois B, Feldman HH, Jacova C, Dekosky ST, Barberger-Gateau P, Cummings J, et al. Research criteria for the diagnosis of Alzheimer's disease: revising the NINCDS-ADRDA criteria. Lancet Neurol. 2007;6(8):734-46.

14. Donohue MC, Gamst AC, Thomas RG, $\mathrm{Xu}$ R, Beckett L, Petersen RC, et al. The relative efficiency of time-to-threshold and rate of change in longitudinal data. Contemp Clin Trials. 2011;32(5):685-93.

15. Donohue MC, Jacqmin-Gadda H, Le Goff M, Thomas RG, Raman R, Gamst $\mathrm{AC}$, et al. Estimating long-term multivariate progression from short-term data. Alzheimers Dement. 2014;10(5 Suppl):S400-10.

16. Dartigues JF, Gagnon M, Barberger-Gateau P, Letenneur L, Commenges D, Sauvel C, et al. The Paquid epidemiological program on brain ageing. Neuroepidemiology. 1992;11 Suppl 1:14-8.

17. Amieva H, Mokri H, Le Goff M, Meillon C, Jacqmin-Gadda H, FoubertSamier A, et al. Compensatory mechanisms in higher-educated subjects with Alzheimer's disease: a study of 20 years of cognitive decline. Brain 2014;137(Pt 4):1167-75.

18. Ayutyanont N, Langbaum JB, Hendrix SB, Chen K, Fleisher AS, Friesenhahn $\mathrm{M}$, et al. The Alzheimer's Prevention Initiative composite cognitive test score: sample size estimates for the evaluation of preclinical Alzheimer's disease treatments in presenilin 1 E280A mutation carriers. J Clin Psychiatry. 2014;in press.

19. Raghavan N, Samtani MN, Farnum M, Yang E, Novak G, Grundman M, et al. The ADAS-Cog revisited: novel composite scales based on ADAS-Cog to improve efficiency in MCI and early AD trials. Alzheimers Dement. 2013;9(1 Suppl):S21-31.

20. Donohue MC, sperling RA, Salmon DP, Rentz DM, Raman R, Thomas RG, et al. The preclinical Alzheimer cognitive composite: measuring amyloidrelated decline. JAMA Neurol. 2014;71(8):961-70.

21. Frank LB. Commentary on «Health economics and the value of therapy in Alzheimer's disease.» Report from the Alzheimer's Association Research Roundtable on patient-reported outcomes and dementia research. Alzheimers Dement. 2007;3(3):162-5.

22. Amariglio RE, Donohue MC, Marshall GA, Rentz DM, Salmon DP, Ferris SH, et al. Tracking early decline in cognitive functionin older individuals at risk for Alzheimer disease dementia: The Alzheimer's Disease Cooperative Study Cognitive Function Instrument. JAMA Neurol. 2015;in press.

23. Liu-Seifert H, Siemers E, Sundell K, Price K, Han B, Selzler K, et al Cognitive and functional decline and their relationship in patients with mild Alzheimer's dementia. J Alzheimers Dis. 2015;43(3):949-55. 